\title{
Physical Activity during Ramadan and COVID-19
}

\author{
Rashidda H Hamid
}

Physiotherapist- FemFit Pune. India - 411048.

\begin{abstract}
Ramadan is a holy month in Islamic calendar where fasting is observed for 29-30 days. Fasting causes a lot of changes in the body's physical activity as food and water intake timings are altered. Physical activity is a key to fight the novel corona virus. Timing of the exercises should be adjusted in a manner that does not lead to severe starvation or dehydration. Fasting in the month of Ramadan during the pandemic is more of a personalised decision.
\end{abstract}

Keywords- Fasting, Ramadan, fitness, Conid-19 fitness.

\section{INTRODUCTION}

Ramadan is the $9^{\text {th }}$ month of the Islamic calendar (lunar month) and the holiest month for Muslims. The duration of Ramadan is of 29-30 days. As it follows the lunar cycle it falls 11 days earlier every year and thereby can fall in any of the 4 seasons. As the time of Ramadan month varies every year the hours of fasting also vary between 11-18 hours depending upon the geographical location. During this month Muslims, all over the world observe fastingabstinence from food, water, smoking, sexual desires from dawn (Suhoor) till dusk (Iftaar). Food and water habits are changed with respect to normal daily routine of 3 meals with supplemental water to 2 meals in Ramadan and supplemental water between dusk to dawn. This change of food pattern is also accompanied with a change of sleeping pattern and reduction of physical activity. ${ }^{[1,}$

Change of food habits and sleep timings affect the body's physical activity, the appetite and the hormonal responses to food. These may also have alterations with biological processes of the body. In literature reported by Nader Lessan et al. it was found that Ramadan fasting has been associated with variable weight changes, ranging from modest weight gain to weight neutrality and weight loss, with a reported reduction in total calorie intake in some, but not all, populations. Weight loss observed in some subjects tends to be regained shortly after Ramadan ${ }^{[5]}$

In 2019, severe respiratory lung diseases were developed due to the novel corona virus called COVID-19. It has been several months since COVID-19 spread and humans have failed to fight this deadly virus successfully Older people with underlying health problems, and/or immunodeficiency, are more likely to have a poor outcome when they are infected with the virus. Given the pandemic situation, scientists, scholars and physicians are unable to give a clear rationale of fasting to be safe to be followed during this pandemic. The decision to observe fasts has been left on the individual with a few points to keep in mind. Here are some preventative suggestions for people who are eager to fasting under the COVID19 pandemic conditions:

- Adherence to the WHO recommendations: this includes frequent hand washing; maintaining a distance of at least one meter from others, and wearing a mask.

- Most religious authorities have always stated that, if a person has problems with 
ill-health, it is better to refrain from fasting.

- During fasting, avoid being in a crowded public space such as bus, subway, etc.

- Have more rest during fasting.

- Avoid going to religious places for prayer and supplication ${ }^{[6]}$

Literature also states that fasting benefits in non-communicable diseases like obesity, heart conditions, diabetes-type 2, reduces insulin resistance, keeps the fat mass in control along with total and LDL cholesterol. White blood cells that rejuvenate the immune system are produced in the body after Fasting for at least 3 days. This helps the immune system to fight infection ${ }^{[6]}$

The US Centers for Disease Control and Prevention (CDC) has identified risk factors for severe COVID-19, including advanced age, sex (male) and the presence of underlying comorbidities, such as diabetes, obesity and cardiovascular disease. [1] Along with this they have also documented that lack of physical activity preceding the pandemic have higher chances of ICU admissions in individuals affected with COVID-19. Physical inactivity was stated to be the strongest risk factor for having severe outcomes in affected with COVID-19. The US Physical Activity Guidelines call for all adults to engage in at least $150 \mathrm{~min} /$ week of moderate to vigorous physical activity ${ }^{[7]}$

A well maintained and boosting immune function and enhancing individual resistance is known to strengthen personal immunity and is essential in fighting COVID-19. A balanced diet along with moderate duration of exercise training of 45-60 minutes is found to improve immune function and enhances functional activity. These bouts of moderate intensity exercises over time add up to an improved cellular immune function ${ }^{[7]}$

Dehydration is a possibility in Ramadan. Therefore exercises should be carried out at a time in which the water stores can be replenished. In literature two times are suggested one is found to be 2-3 hours before iftaar. Any dehydration suffered when exercising at this time can be replenished after iftaar. The second appropriate time to exercise in Ramadan is 2-3 hours after iftaar. At this time the food is digested energy stores are replenished and body can actively exercise without a scare of dehydration ${ }^{[8]}$

Exercising in Ramadan benefits the body by maintain the strength and endurance, metabolism, prevents muscle breakdown. It also benefits the soul by being active and less tried and helps to perform the salah prayers and rituals with vigor. Literature suggests to follow a basic exercise program of warm up, aerobic exercises, resistance training, stretching exercises and cool down while fasting during Ramadan. The time dedicated to exercises should be 150 minutes per week ${ }^{[8]}$

Warm up is essential for the body for a gradual increase in body temperature and avoiding muscle injuries thus should be followed on all days of exercises at $40-50 \%$ of HR Max for 7-10 minutes. Aerobic training of $50-75 \% \mathrm{Hr}$ Max for $15-20$ minutes. Aerobic exercises have known to mobilise fats and keep one active. Resistance training at $50-75 \%$ of $1 \mathrm{RM}$ for 15-20 minutes. To maintain and improve muscle strength and glucose uptake by muscles. Stretching exercises and a cool down of minimum 5 minutes at $40-50 \%$ HR Max is very essential component of the exercise protocol to prevent sudden syncope and blood pressure irregularities. Stretching exercises helps to stretch the muscles and maintain flexibility. Exercises should be carried out in a cool and well ventilated environment for maximum benefit ${ }^{[7,9]}$

Thus, Fasting during Ramadan in the pandemic time is more an individualised decision taken as per ones belief. Being physically fit is of utmost importance to stay healthy during the holy month and fighting the virus.

Acknowledgement: None 


\section{Conflict of Interest: None}

\section{Source of Funding: None}

\section{REFERENCES}

1. Trabelsi, Zeghal et al. Effect of Resistance Training During Ramadan on Body Composition and Markers of Renal Function, Metabolism, Inflammation, and Immunity in Recreational Bodybuilders. International Journal of Sport Nutrition and Exercise Metabolism, 2012; 22: 267 -275

2. Mohsen Mazidi et al. The Effects of Ramadan Fasting on Body Composition. Shiraz E-Med J. January 2014; 15(1)

3. Naif S. Al-barha and Khalid S. Aljaloud. The Effect of Ramadan Fasting on Body Composition and Metabolic Syndrome in Apparently Healthy Men. American Journal of Men's Health. 2018.

4. Ziaee $\mathrm{V}$ et al. The changes of metabolic profile and weight during Ramadan fasting. Islamic Singapore Med J 2006; 47(5): 409.

5. Nader Lessan et al. The effects of Ramadan fasting on activity and energy expenditure.
Am J Clin Nutr 2018;107:54-61. Printed in USA. 2018 American Society for Nutrition.

6. Majid Taati Moghadam et al. Ramadan Fasting During the COVID-19 Pandemic; Observance of Health, Nutrition and Exercise Criteria for Improving the Immune System. 2021. Front. Nutr. 7:570235

7. Sallis R, et al. Physical inactivity is associated with a higher risk for severe COVID-19 outcomes: a study in 48440 adult patients. British Journal of Sports Medicine. 2021.

8. FIND. Nutrition during the holy month of Ramadan. Special focus on management of infectious diseases. Volume 1.

9. Carolyn Kisner, Lynn Allen Colby. Exercise Program. In: Therapeutic exercises foundations and techniques. $6^{\text {th }}$ Ed.By. F A Davis Company Philadelphia 2012. P 250251.

How to cite this article: Hamid RH. Physical activity during Ramadan and COVID-19. Int J Health Sci Res. 2021; 11(5):125-127. DOI: https://doi.org/10.52403/ijhsr.20210517 